\title{
Hole Diameter ratio (HDR) for prediction of anatomical outcomes in stage III and IV idiopathic macular holes
}

\section{Yue Qi}

Beijing Tongren Hospital

\section{Yanping Yu}

beijing Tongren hospital

\section{Zengyi Wang}

Beijing Tongren Hospital

\section{Jing Wang}

Beijing Tongren Hospital

\section{Wu Liu ( $\nabla$ wuliubj@sina.com )}

Department of Ophthalmology, Beijing Tongren Eye Center, Beijing Tongren Hospital, Capital Medical University, Beijing Ophthalmology and Visual Sciences Key Lab, Beijing, China https://orcid.org/00000001-7967-6924

\section{Research article}

Keywords:

Posted Date: June 1st, 2020

DOI: https://doi.org/10.21203/rs.3.rs-29158/v1

License: (9) (1) This work is licensed under a Creative Commons Attribution 4.0 International License. Read Full License

Version of Record: A version of this preprint was published on August 28th, 2020. See the published version at https://doi.org/10.1186/s12886-020-01614-7. 


\section{Abstract}

Summary: In this study, we reviewed 101 eyes with stage III or IV IMHs treated by PPV combined with ILM peeling and room air tamponade, took the ratio of minimum diameter to maximum diameter (hole diameter ratio, $\mathrm{HDR}$ ) as the predictive factor and found $\mathrm{HDR}<0.6$ (" $\mathrm{A}$ " type $\mathrm{IMH}$ ) can be a predictive factor for a good anatomical outcome.

Purpose: To identify hole diameter ratio (HDR) as the predictive factor for unclosed stage III and IV idiopathic macular holes (IMHs) by the use of preoperative optical coherence tomography (OCT).

Methods: 101 eyes with stage III or IV IMH were included in this retrospective case series study. \All cases were treated with vitrectomy combined with ILM peeling and room air tamponade. The MH minimum and maximum diameter, the ratio of the minimum to the maximum diameter (which defined as HDR) were performed.

Results: 81 eyes (80.2\%) got a Type I closure after surgery (group A). Postoperative unclosed MHs were found in 20 eyes (19.8\%) (group B). The minimal diameter of IMHs (703.6 \pm 116.1 um VS 597.6 \pm 120.1 um, $P<0.01)$ and HDR $(0.6 \pm 0.1$ VS $0.5 \pm 0.1, P=0.01)$ were both significantly different between two groups. The cutoff value of the receiver operating characteristic curve was 0.6 . The IMH with the HDR that less than 0.6 were defined as " $\mathrm{A}$ " type IMH and the others were defined as " $\mathrm{H}$ " type IMH. The closure rate of two type IMHs were $90.2 \%$ and $65.0 \%$ respectively. It had significant differences. $(P=0.002)$

Conclusions: HDR<0.6("A" type IMH) can be a predictive factor for a good anatomical outcome in stage III and IV IMHs after vitrectomy combined with LM peeling and room air tamponade.

\section{Background}

Idiopathic macular hole (IMH) is one of the most often causes of poor vision. The estimated incidence of $\mathrm{IMH}$ ranged from $0.3-0.8 \%$ in the population. ${ }^{1,2}$ It may cause a small dark spot in the central vision. $\mathrm{MH}$ may be related with high myopia or ocular trauma, but the reason of most MHs is unknown (idiopathic). ${ }^{3}$ Based on the optical coherence tomography (OCT) images of macular region, IMHs can be divided into 4 stages, stage III or IV MH was taken as refractory $\mathrm{MH}$ for the low close rate after surgery. ${ }^{4}$

Pars plana vitrectomy (PPV) is the most popular way to treat full-thickness $\mathrm{MH}$ at present. ${ }^{5}$ Internal limiting membrane (ILM) peeling has been proved to increase the closure rates of IMHs. ${ }^{6}$ More than $90 \%$ of IMHs were closed after PPV combined with ILM peeling and inert gas or room air tamponade. ${ }^{7-10}$ The initial closure rates of IMHs ranged from $75.6-100 \% .{ }^{11-15}$ However, there are still some IMHs cannot be healed after surgery, especially in stage III and IV IMHs. The reason is still unknown.

In clinical practice, most unclosed IMHs were stage III and IV IMHs, especially when PPV combined with the short-term gas-room air. Therefore, the preoperative factors that affect the closure of stage III and IV IMHs are particularly important. It can help prompting surgeons to choose different operation methods 
before operation. Several studies have demonstrated the indexes according preoperative OCT features to predict the anatomic results after surgical interventions for MHs. While quantity of these studies and sizes of their samples were relatively small and none of them were aimed specially at stage III or IV IMHs which are the hardest IMHs to be healed. These parameters are also complex and difficult to calculate. 16-19 It's not that fit for the busy clinical work to make a quick judgement.

In this study, we retrospectively reviewed the records of 101 eyes with stage III and IV IMHs and took the ratio of minimum diameter to maximum diameter (we named it as hole diameter ratio, HDR) as the predictive factor to predict the anatomical outcomes. All of them were treated with PPV combined with ILM peeling and room air tamponade.

\section{Methods}

This study followed the tenets of the Declaration of Helsinki and was approved by the Institutional Review Board of the Beijing Tongren Hospital, Capital Medical University. It was a retrospective, interventional case series study. Patients with stage III or IV MHs who underwent PPV with ILM peeling and room air tamponade at Beijing Tongren Eye Center from November, 2016 to September, 2017 and July, 2018 to November, 2018 were included. Before the surgical procedure, informed consent was obtained from all patients.

Patients less than fifty years old, or with a stage I and II IMH ${ }^{4}$, or high myopia, or history of vitrectomy, or any other fundus diseases will be excluded.

The data of age, sex, preoperative lens status, refractive error, axial length, preoperative best-corrected visual acuity (BCVA), and OCT findings were recoded. The IMHs' minimum and maximum diameter, HDR, were measured and calculated

OCT (Carl Zeiss, Dublin, CA, USA) was performed pre- and post-operation. The OCT images were reviewed and analyzed independently by two separate investigators (Y.Q. and YP.Y.). Any discrepancies were resolved by discussion with the principal investigator (W.L.) until a consensus was reached.

A standard 23-gauge 3-port pars plana vitrectomy was performed by the same experienced surgeon (W.L.). Phacoemulsification and IOL implantation were first performed if necessary. Vitrectomy was performed and the posterior hyaloid was elevated and trimmed in all patients. Then the ILM was peeled off with forceps in an area of at least 2disc diameter around the $\mathrm{MH}$ without staining. The vitreous was filled with room air at the end of the surgery. Patients were asked to stay in a prone position for at least 5 days after surgery. At postoperative follow-up examinations, measurements of visual acuity and OCT were performed at 2-4 weeks. No serious postoperative complications happened.

Statistical analysis was performed using SPSS for Windows (version 23.0; IBM-SPSS, Chicago, IL). The results were expressed as the mean \pm standard deviation (SD). Differences in the incidence rates of OCT findings between patients with or without unclosed $\mathrm{MH}$ were analyzed by corrected Chi-square test. The 
measurements of BCVA were converted into the logMAR (logarithm of the minimum angle of resolution). Mean age, duration of symptoms, axial length, BCVA, intraocular pressure and refractive error were analyzed by independent samples t-test. The discriminating power of HDR to predict closure rate after initial surgery was estimated by calculating the area under the receiver operating characteristic curve, with the area larger than 0.6 considered not determined by chance. Normal distributions of the data were checked before selecting the statistical analysis methods. A P-value $<0.05$ was considered statistically significant.

\section{Results}

\section{Preoperative Clinical Characteristics}

There were 101 eyes of 101 patients with stage III $(n=65,64.4 \%)$ or IV $(n=36,35.6 \%)$ IMH that underwent PPV combined with ILM peeling and air tamponade. 26 patients $(25.7 \%)$ were men, and 75 patients (74.3\%) were women. One eye (1.0\%) was pseudophakic and 100 eyes (99.0\%) were phakic.

\section{Comparison of the Preoperative Clinical Characters of the Two Groups}

81 eyes (80.2\%) got a closed IMH (Type I) after one operation (defined as Group A). Postoperative unclosed IMHs were found in 20 eyes (19.8\%) (defined as Group B). There were 50 (61.7\%) stage III IMHs and $31(38.3 \%)$ stage IV IMHs in group A and $15(75.0 \%)$ stage III IMHs and $5(25.0 \%)$ stage IV IMHs in group $B$. Patients in group $A$ and $B$ did not differ significantly (all $P>0.05)$ in age $(64.1 \pm 4.4$ years versus $67.0 \pm 4.5$ years; $P=0.32)$, duration of symptoms $(8.3 \pm 11.1$ months versus $8.3 \pm 3.9$ months, $P=0.38)$, preoperative BCVA $(1.1 \pm 0.4 \log$ MAR versus $1.0 \pm 0.4 \log M A R, P=0.68)$, preoperative IOP $(15.5 \pm$ $2.7 \mathrm{mmHg}$ versus $16.0 \pm 3.5 \mathrm{mmHg}, P=0.46)$, axial length $(23.4 \pm 0.7 \mathrm{~mm}$ versus $22.9 \pm 0.7 \mathrm{~mm} ; P=0.24)$ (Tables 1). 
Table 1

Comparison of Pre-operative Characteristics in Two Groups

\begin{tabular}{|lllll}
\hline & Group A & Group B & t & P \\
\hline Eyes & 81 & 20 & & \\
\hline Age (years) & $64.1 \pm 4.4$ & $67.0 \pm 4.5$ & -0.9 & 0.32 \\
\hline Duration of symptoms (months) & $8.3 \pm 11.1$ & $8.3 \pm 3.9$ & -0.9 & 0.38 \\
\hline PreopBCVA(LogMAR) & $1.1 \pm 0.4$ & $1.0 \pm 0.4$ & -0.4 & 0.68 \\
\hline IOP (mmHg) & $15.5 \pm 2.8$ & $16.0 \pm 3.5$ & 0.2 & 0.46 \\
\hline Axial Length(mm) & $23.4 \pm 0.7$ & $22.9 \pm 0.7$ & 0.2 & 0.24 \\
\hline Minimum Diameter (um) & $597.6 \pm 120.1$ & $703.6 \pm 116.1$ & -3.6 & $<0.01$ \\
\hline HDR & $0.5 \pm 0.1$ & $0.6 \pm 0.1$ & -1.9 & 0.03 \\
\hline Group A: eyes with closed MH; Group B: eyes with unclosed MH; & & \\
\hline BCVA: best corrected visual acuity; IOP: intra-ocular pressure; & & \\
\hline
\end{tabular}

Minimum diameter of IMH was also analyzed. The mean minimum diameter was $597.6 \pm 120.1 \mathrm{um}$ in group $A$ and $703.6 \pm 116.1$ um in group $B$. It had significant differences between two groups $(t=-3.6, P<$ $0.01)$.

\section{Analysis of HDR in Two Groups}

The mean HDR was $0.5 \pm 0.1$ in group A and $0.6 \pm 0.1$ in group $B$. It was significantly different in two groups $(\mathrm{t}=-1.9 \mathrm{P}=0.03)$.

The area under the receiver operating characteristic curve was 0.7 (95\% confidence interval $0.6-0.8)$ for HDR with a cutoff value of 0.6 , a sensitivity of $75.0 \%$, and a specificity of $67.9 \%$ for predicting closure rate after initial surgery $(P=0.01$, shown as Fig. 1$)$.

For the cutoff value was 0.6 (which got from the receiver operating characteristic curve), we defined IMHs with the HDR that less than 0.6 as "A" type IMHs and IMHs with the HDR that more than or equal to 0.6 as "H" type IMHs. There were 55 "A" type IMHs (67.9\%) in group A and 6 "A" type IMHs (30.0\%) in group B, and 26 " $\mathrm{H}$ " type IMHs (32.1\%) in group A and 14 " $\mathrm{H}$ " type IMHs (70.0\%) in group B. Two types of IMHs were significantly different in two groups $\left(X^{2}=9.6, P=0.002\right)$.

The mean diameter had significant differences between " $\mathrm{A}$ " and " $\mathrm{H}$ " type IMHs ( $567.0 \pm 108.2$ um versus $697.3 \pm 110.9 u m, t=-5.9, P<0.01)$. The diameter of two type IMHs were significantly different in group $A$ (559.0 \pm 102.9 um versus $679.1 \pm 114.6$ um, $t=-4.7, P<0.01)$, while not in group $B(639.7 \pm 137.5$ um versus $731.0 \pm 98.7 \mathrm{um}, \mathrm{t}=-1.7, \mathrm{P}=0.11)$. For "A" type IMHs, both the mean diameter (559.0 \pm 102.9 um versus 
$639.7 \pm 137.5 \mathrm{um}, \mathrm{t}=-1.8 ; \mathrm{P}=0.1)$ and $\mathrm{HDR}(0.5 \pm 0.1$ versus $0.5 \pm 0.02, \mathrm{t}=-0.9, \mathrm{P}=0.4)$ were not significantly different between group $A$ and $B$. Also, for " $\mathrm{H}$ " type IMHs, the mean diameter (679.1 \pm 114 .6um versus $731.0 \pm 98.7 \mathrm{um}, \mathrm{t}=-1.4 ; \mathrm{P}=0.2)$ and $\mathrm{HDR}(0.6 \pm 0.1$ versus $0.6 \pm 0.04, \mathrm{t}=1.0, \mathrm{P}=0.3)$ had no significantly differences between group $A$ and $B$. (Table 2)

Table 2

Comparation of two type IMHs between group A and B

\begin{tabular}{|c|c|c|c|c|c|}
\hline & & $\begin{array}{l}\text { A Type } \\
\text { IMH }\end{array}$ & $\begin{array}{l}\text { H Type } \\
\text { IMH }\end{array}$ & $\mathrm{t}$ & $\mathbf{P}$ \\
\hline \multirow[t]{3}{*}{ Group A } & $\mathrm{N}(\%)$ & $55(67.9)$ & $26(32.1)$ & & \\
\hline & Diameter(um) & $\begin{array}{l}559.0 \pm \\
102.9\end{array}$ & $\begin{array}{l}679.1 \pm \\
114.6\end{array}$ & -4.7 & 0.000 \\
\hline & HDR & $0.5 \pm 0.1$ & $0.6 \pm 0.1$ & & \\
\hline \multirow[t]{3}{*}{ Group B } & $\mathrm{N}(\%)$ & $6(30)$ & $14(70)$ & & \\
\hline & Diameter(um) & $\begin{array}{l}639.7 \pm \\
137.5\end{array}$ & $\begin{array}{l}731.0 \pm \\
98.7\end{array}$ & -1.7 & 0.11 \\
\hline & HDR & $0.5 \pm 0.02$ & $0.6 \pm 0.04$ & & \\
\hline Mean Diameter (um) & & $\begin{array}{l}567.0 \pm \\
108.2\end{array}$ & $\begin{array}{l}697.3 \pm \\
110.9\end{array}$ & -5.9 & 0.000 \\
\hline \multirow{2}{*}{$\begin{array}{l}\text { Comparation of Diameter between group } \\
A \text { and } B\end{array}$} & $\mathrm{t}$ & -1.8 & -1.4 & & \\
\hline & $P$ & 0.1 & 0.2 & & \\
\hline \multirow{2}{*}{$\begin{array}{l}\text { Comparation of HDR between group A } \\
\text { and } B\end{array}$} & $\mathrm{t}$ & -0.9 & 1.0 & & \\
\hline & $P$ & 0.4 & 0.3 & & \\
\hline \multicolumn{6}{|l|}{ IMH: idiopathic macular hole; } \\
\hline HDR: Hole diameter ratio; & & & & & \\
\hline
\end{tabular}

\section{Comparison of closure rate of two types of IMHs}

There were 61 eyes with "A" type IMHs and 40 eyes with " $H$ " type IMHs. In eyes with "A" type IMHs, 55 of 61 eyes got closed MHs following one surgical procedure, and the anatomical success rate was $90.2 \%$. While in eyes with " $\mathrm{H}$ " type IMHs, only 26 of 40 eyes got closed MHs, and the anatomical success rate was $65.0 \%$. The closure rates of two types of IMHs were significantly different. $(P<0.01)$

\section{Discussion}


In this study, we identified HDR as a predictive factor for unclosed IMHs after PPV combined with ILM peeling in stage III and IV IMHs and found the IMH with HDR more than 0.6 ("H" type IMH) was hard to heal.

The relationships between preoperative OCT measurements and prognosis were revealed for the first time by Ip et al. at 2002. ${ }^{16}$ By comparing the anatomical reduction rate and recurrence rate of the IMH after operation, they found that IMH with a diameter under 400 um had a higher recovery rate. Kusuhara et al. 18 proposed the concept of macular hole index ( $\mathrm{MHI}$, ratio of hole height to base diameter of hole) and found that MHI significantly correlated with the postoperative BCVA. While, most of the current studies associated with $\mathrm{MHI}$ are limited to the IMHs under stage III. Hole form factor (HFF) ${ }^{20}$ defined as the ratio of the sum of the lengths of the left and right oblique sides to the base diameters of the IMH. Ullrich et al. 21 found in patients with HFF $>0.9$ the macular hole was closed following one surgical procedure, whereas in eyes with HFF $<0.5$ anatomical success rate was only $67 \%$. Haritoglou et al. ${ }^{22}$ believed that higher HFF correlated with better postoperative visual outcome and the correlation coefficient is 0.36 . Ruiz-Moreno et al. ${ }^{19}$ proposed tractional hole index (THI, ratio of height to minimum diameter) and diameter hole index ( $\mathrm{DHI}$, ratio of minimum diameter to baseline diameter). They found THI correlated significantly with postoperative best spectacle corrected visual acuity, but DHI did not. Wakely L et al. ${ }^{23}$ measured macular hole inner opening diameter, minimum linear diameter, base diameter and macular hole height and calculated the MHI and FHI. They found base diameter, macular hole inner opening and minimum linear diameter could be used to predict anatomical and/or functional success rate of macular hole surgery. Preoperative base diameter is the most useful variable in this regard. Mingwei Zhao et al. ${ }^{24}$ proposed a method named macular hole closure index $(\mathrm{MHCl})$ to predict anatomical outcome after IMH surgery. $\mathrm{MHCl}$ was calculated as $(\mathrm{M}+\mathrm{N})$ /BASE based on the preoperative OCT status. $\mathrm{M}$ and $\mathrm{N}$ were the curve lengths of the detached photoreceptor arms, and BASE was the length of the retinal pigment epithelial layer (RPE layer) detaching from the photoreceptors. Some studies used central subfield retinal thickness (CSRT) to predict anatomical results of MH surgery. While these results were still contradictory. ${ }^{25,26}$

All these indexes can predict the anatomical or functional outcomes of IMH surgery in certain degrees. While they were either complex in calculation or lack of convincingness for obtained from a small sample. It is known to all that stage III and IV IMHs are more difficult to heal ${ }^{4}$. While few articles have been devoted to factors that affecting the healing of stage III and IV IMHs. In this study, we reviewed the records of 101 eyes treated by PPV combined with ILM peeling and room air tamponade to determine the anatomical predictive factors for stage III and IV IMHs and tried to find an easy way to evaluate it before surgery.

In this study, there were no significant differences in age, duration of symptoms, preoperative BCVA, intraocular pressure (IOP) and axial length between patients with and without unclosed IMHs. While the minimal diameter $(P<0.01)$ and the HDR $(P=0.01)$ are significantly different in two groups. Which means the minimum diameter and HDR are two important factors for closure of stage III and IV IMHs. 
Diameter is an important factor to influence the result of IMH surgery. It has been reported that IMH with a minimum diameter under 400um had a higher recovery rate. ${ }^{6}$ Wu Liu et al. ${ }^{11}$ found the diameter more than 677um is a risk factors for stage III and IV IMH that underwent 23-gauge vitrectomy, ILM peeling, and air tamponade. In this study, the mean minimum diameter was 597.57.09 \pm 120.14 um in eyes with closed stage III or IV IMHs and $703.60 \pm 116.13$ um in eyes with unclosed stage III or IV IMHs $(P<0.01)$. Which means the minimum diameter is indeed an important factor for postoperative anatomical outcomes in stage III and IV IMHs. The result in these cases is consistent with previous literature.

Based on past experiences, we found the forms were different between eyes with closed and unclosed stage III/IV IMH, thus we tried to find a method to predict the closure of stage III and IV IMHs though the IMH's shape before surgery and hoped that this method is simple and easy to use in clinical work.

HDR (hole diameter ratio) is defined as minimum diameter to maximum diameter in this study. It reflects the magnitude of pulling force in the tangential direction of the IMH. The mean HDR was $0.5 \pm 0.1$ in eyes with closed IMHs and $0.6 \pm 0.1$ in eyes with unclosed IMHs. It was significantly different in two groups ( $P$ = 0.03). Ratio of minimum diameter to maximum diameter has been mentioned as diameter hole index (DHI) in previous studies. While in those study, no relationship was found between the ratio and anatomical/functional outcome. We think the reasons for the difference conclusions may be as follow: First, the objects were different. All stages of IMHs were included in previous studies, while only stage III and IV IMHs were included in this study. Stage III and IV IMHs are refractory IMHs. The diameters are larger and the pulling force in the tangential direction may play a more important role in these IMHs (In stage III/IV IMHs, posterior vitreous detachment happens, which means the tangential force become the main force to IMH). Therefore, HDR may be more suitable for evaluating stage III/IV macular holes. Second, sample size is different. In previous studies, the sample size was mostly below 50 , which was relatively small. The sample size of this study is more than 100 , and the conclusion is relatively more accurate. Also, the tamponades, the surgeon and others can all be factors that influence the conclusions.

As HDR with a cutoff value of 0.6 in these cases, we chose 0.6 as a dividing line to defining different types of IMHs. The IMHs with the HDR that less than 0.6 were defined as " $A$ " type IMHs. The IMHs with the HDR that more than or equal to 0.6 was defined as " $\mathrm{H}$ " type IMHs. We found the mean diameter had significant differences between " $A$ " and " $H$ " type IMHs $(P<0.01)$ in this study, which means " $H$ " type IMHs are with larger diameter. (Table 2) This indicates that the HDR itself contains the element of diameter.

The closure rate of stage III/IV IMHs is $90.2 \%$ in "A" type IMHs and only $65.0 \%$ in "H" type IMHs. It had significant differences in type of IMHs $(P=0.002)$. Which means the anatomical results can be well predicted according HDR. We classified IMHs according to HDR and named them according to the shape of IMHs. In this way, the possible prognosis can be judged simply and quickly according to the types of IMH before operation. An "A" type stage III and IV IMH with small diameter is relatively easy to close. While for large IMH with a "H" type shape (HDR greater-than-equal 0.6), we recommend that more methods should be used during surgery. (Fig. 2,3) 
The limitations of the current study included as follow: First, it is a retrospective case series study; Second, we only analyzed the relationship between HDR and anatomical outcomes, the functional outcomes need further discuss.

In conclusion, $\mathrm{HDR}<0.6$ ("A" type IMH) can be a predictive factor for a good anatomical outcome in stage III and IV IMHs after vitrectomy combined with LM peeling and air tamponade. It can be easily calculated and intuitively noticed from OCT and may help doctors and patients predicting the surgical results of stage III and IV MHs.

\section{Abbreviations}

HDR: hole diameter ratio; PPV: pars plana vitrectomy; ILM: internal limiting membrane; IMH: idiopathic macular hole; BCVA: best-corrected visual acuity; OCT: optical coherence tomography; IOL: intra ocular lens; SD: standard deviation; logMAR: logarithm of the minimum angle of resolution.

\section{Declarations}

\section{Ethics approval and consent to participate}

All of the patients gave a written informed consent and the study was conducted in accordance with the tenets of Declaration of Helsinki and approved by the Ethics Committee of Beijing Tongren Hospital (No. 2017-056-02). All procedures performed in studies involving human participants were in accordance with the ethical standards of the institutional and national research committee and with the 1964 Helsinki declaration and its later amendments or comparable ethical standards.

\section{Consent to publish}

Not applicable

\section{Availability of data and materials}

The datasets used and/or analyzed during the current study are available from the corresponding author on reasonable request.

\section{Competing interests}

The authors declare that they have no competing interests.

\section{Funding}




\section{Authors' Contributions}

YQ and WL conceived and designed research; YQ, ZYW, YPY and JW collected data and conducted research; $Y Q$ and $Z Y W$ analyzed and interpreted data; $Y Q$ wrote the initial paper; WL, YPY and YQ revised the paper; WL had primary responsibility for final content. All authors read and approved the final manuscript.

\section{Acknowledgements}

Not applicable.

\section{Reference}

1. Liu L, Yue S, Wu J, Zhang J, Lian J, Huang D, Teng W, Chen L. The Prevalence and Distribution of Vitreoretinal Interface Abnormalities among Urban Community Population in China. J Ophthalmol. 2015; 2015: 742686.

2. Klein R, Klein BE, Wang Q, Moss SE. The epidemiology of epiretinal membranes. Transactions of the American Ophthalmological Society. 1994; 92:403-25.

3. Parravano M, Giansanti F, Eandi CM, Yap YC, Rizzo S, Virgili G. Vitrectomy for idiopathic macular hole. Cochrane Database Syst Rev. 2015; 12;(5):CD009080.

4. Reappraisal of biomicroscopic classification of stages of dev-elopment of a macular hole. Am J Ophthalmol. 1995 Jun;119(6):752-9.

5. Kelly NE, Wendel RT. Vitreous surgery for macular holes. Results of a pilot study. Arch Ophthalmol. 1991; 109:654-659.

6. Brooks HL., Jr Macular hole surgery with and without internal limiting membrane peeling. Ophthalmology. 2000; 107:1939-1949.

7. Kim SS, Smiddy WE, Feuer WJ, Shi W. Outcomes of sulfur hexafluoride (SF6) versus perfluoropropane (C3F8) gas tamponade for macular hole surgery. Retina 2008;28:1408-1415.

8. Briand S, Chalifoux E, Tourville E, et al. Prospective random- ized trial: outcomes of SF6 versus C3F8 in macular hole surgery. Can J Ophthalmol 2015;50:95-100.

9. Essex RW, Kingston ZS, Moreno-Betancur M, et al. The effect of postoperative face-down position and of long- versus short- acting gas in macular hole surgery: results of a registry-based study. Ophthalmology 2016;123:1129-1136.

10. Usui H, Yasukawa T, Hirano Y, et al. Comparative study of the effects of room air and sulfur hexafluoride gas tamponade on functional and morphological recovery after macular hole surgery: a retrospective study. Ophthalmic Res 2013;50:227-230. 
11. Yu Y, Liang $X$, Wang $Z$, et al. Internal limiting membrane peeling AND air tamponade for stage III AND stage IV idiopathic macular holes. Retina. 2018 Oct 3. doi: 10.1097. [Epub ahead of print]

12. Eckardt $\mathrm{C}$, Eckert T, Eckardt $\mathrm{U}$, et al. Macular hole surgery with air tamponade and optical coherence tomography-based duration of face-down positioning. Retina 2008;28:1087-1096.

13. Hejsek L, Stepanov A, Dusova J, et al. Microincision 25G pars plana vitrectomy with peeling of the inner limiting membrane and air tamponade in idiopathic macular hole. Eur $\mathrm{J}$ Ophthalmol 2017;27:93-97.

14. Hasegawa Y, Hata Y, Mochizuki Y, et al. Equivalent tamponade by room air as compared with $S F(6)$ after macular hole surgery. Graefes Arch Clin Exp Ophthalmol 2009;247:1455- 1459.

15. He F, Yu WH, Zheng L, Dong FT. Changes of photoreceptor layer on spectral domain optical coherence tomography in idiopathic macular hole after vitreous surgery combined with air tamponade. Chin J Ophthalmol 2012;48:1088-1092.

16. Ip MS, Baker BJ, Duker JS, et al. Anatomical outcomes of surgery for idiopathic macular hole as determined by optical coherence tomography. Arch Ophthalmol 2002;120(1):29-35.

17. Chhablani J, Khodani M, Hussein $\mathrm{A}$, et al. Role of macular hole angle in macular hole closure. $\mathrm{Br} \mathrm{J}$ Ophthalmol 2015; 99 (12):1634-1638.

18. Kusuhara S, Teraoka Escano MF, Fujii S, et al. Prediction of postoperative visual outcome based on hole configuration by optical coherence tomography in eyes with idiopathic macular holes. Am J Ophthalmol 2004;138(5):709-716.

19. Ruiz-Moreno JM, Staicu C, Pinero DP, et al. Optical coherence tomography predictive factors for macular hole surgery outcome. Br J Ophthalmol 2008; 92(5):640-644.

20. Desai VN, Hee MR, Puliafito CA. Optical coherence tomography of macular holes. In: Madreperla SA, McCuen BW, eds. Macular hole: pathogenesis, diagnosis and treatment. Oxford: ButterworthHeinemann 1999:37-47.

21. Ullrich S, Haritoglou C, Gass C, et al. Macular hole size as a prognostic factor in macular hole surgery. Br J Ophthalmol 2002; 86 (4): 390-393.

22. Haritoglou C, Neubauer AS, Reiniger IW, et al. Long-term functional outcome of macular hole surgery correlated to optical coherence tomography measurements. Clin Exp Ophthalmol 2007; 35 (3): 208213.

23. Wakely L, Rahman R, Stephenson J. A comparison of several methods of macular hole measurement using optical coherence tomography, and their value in predicting anatomical and visual outcomes. Br J Ophthalmol 2012; 96(7): 1003-1007.

24. Liu P, Sun Y, Dong C, et al. A new method to predict anatomical outcome after idiopathic macular hole surgery. Graefes Arch Clin Exp Ophthalmol 2016;254(4): 683-688.

25. Shpak AA, Shkvorchenko DO, Sharafetdinov I, et al. Predicting anatomical results of surgical treatment of idiopathic macular hole. Int J Ophthalmol 2016; 9(2): 253-257. 
26. Jian-Ping Gao, Xiao-Jian Guo.Influences of OCT image on the prognosis of visual outcomes among patients with idiopathic macular holes. Guo Ji Yan Ke Za Zhi. 2013; 13(11): 2293-2295.

\section{Figures}

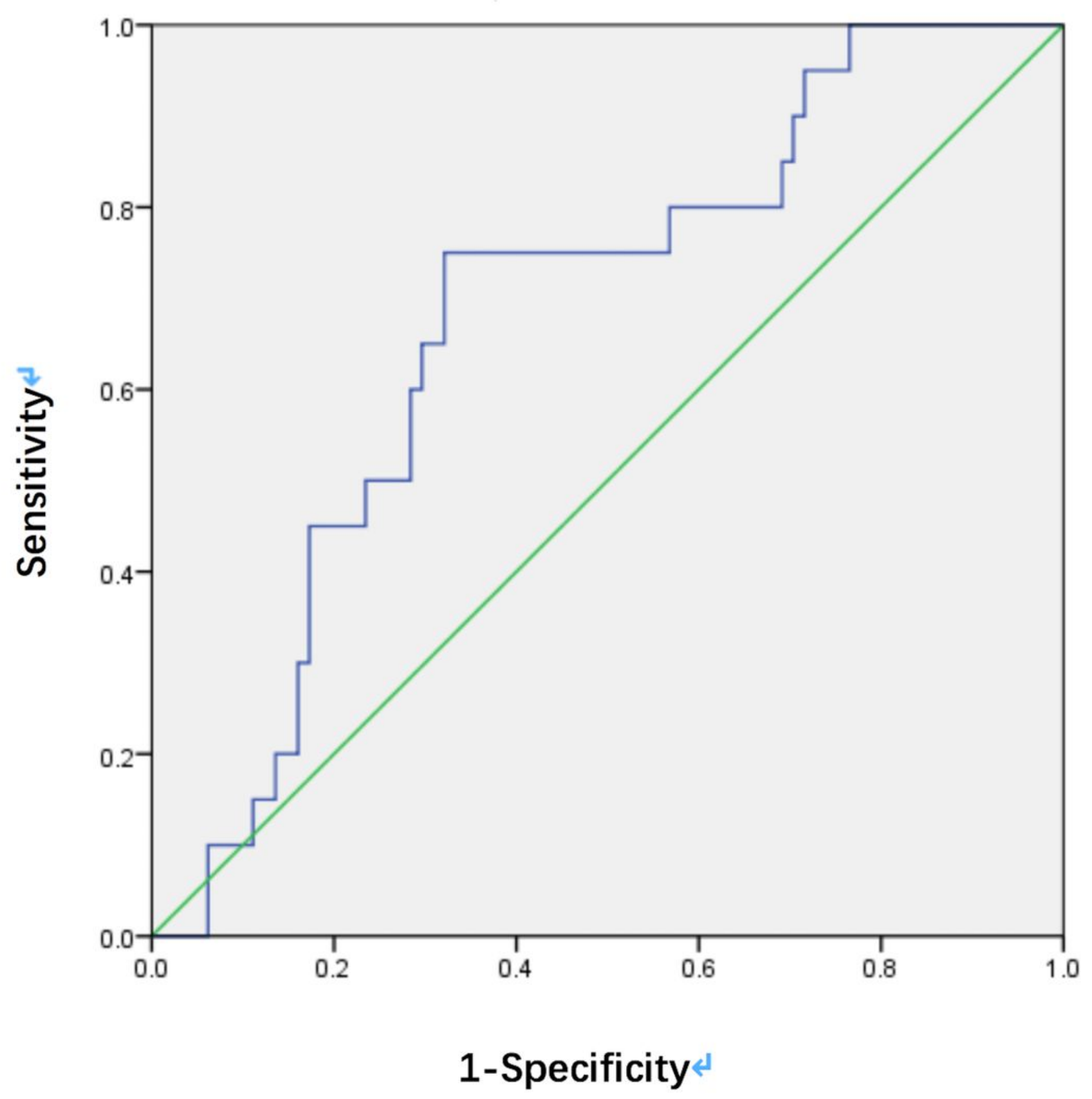

Figure 1

The receiver operating characteristic curve for HDR of $\mathrm{MH}$ predicting initial closure. The area under the curve was 0.7 (95\% confidence interval 0.6-0.8) with a cutoff value of 0.6. Predicting sensitivity and specificity were $75.0 \%$ and $67.9 \%$, respectively $(P=0.01)$. 


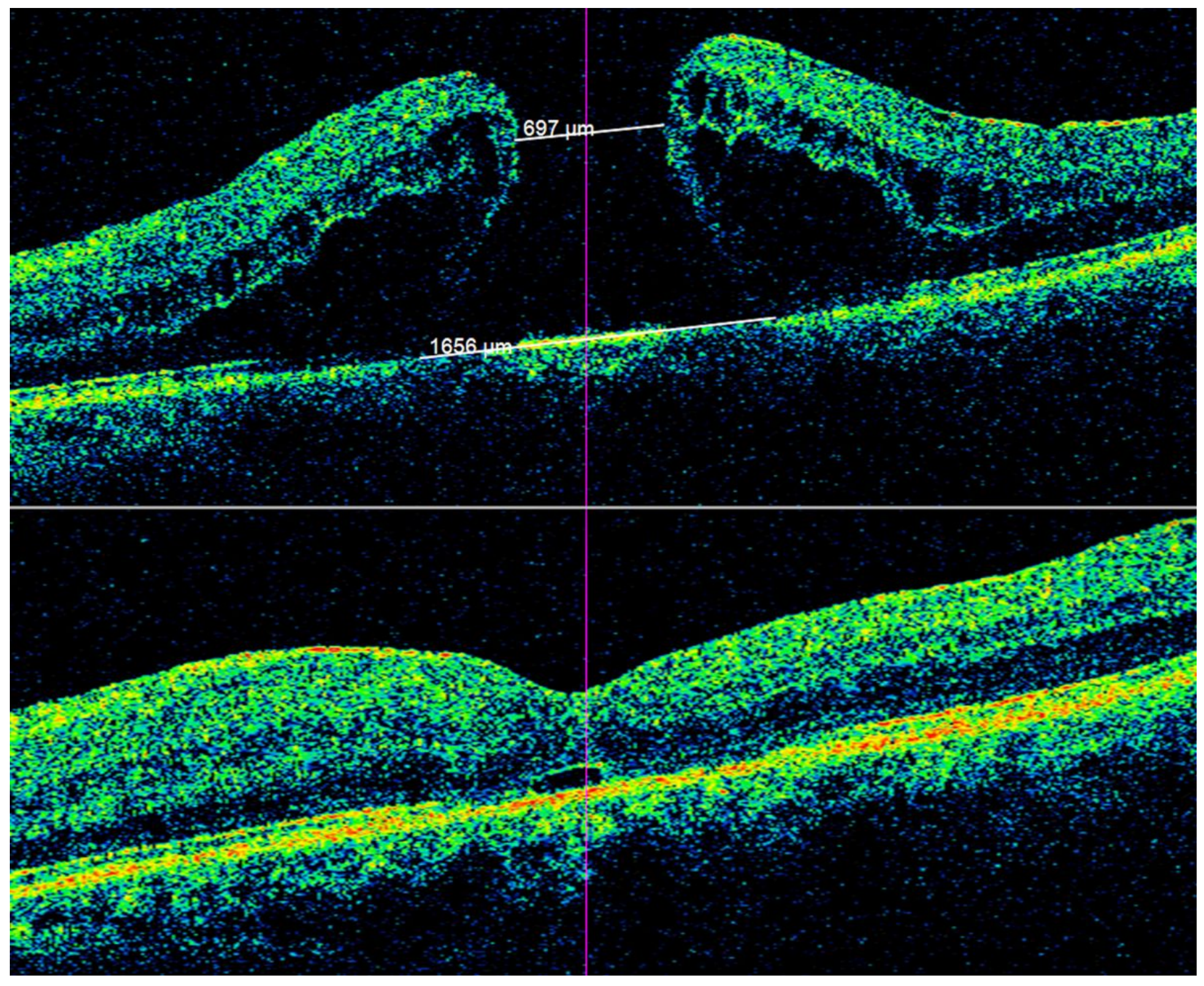

Figure 2

This is a 63-year-old man with an " $\mathrm{A}$ " type IMH. The minimal diameter was 697um and maximum diameter was 1656um. The diameter of his MH was "large" but the HDR was less than 0.6. The MH was closed 1 month after PPV combined with ILM peeling and room air tamponade. 


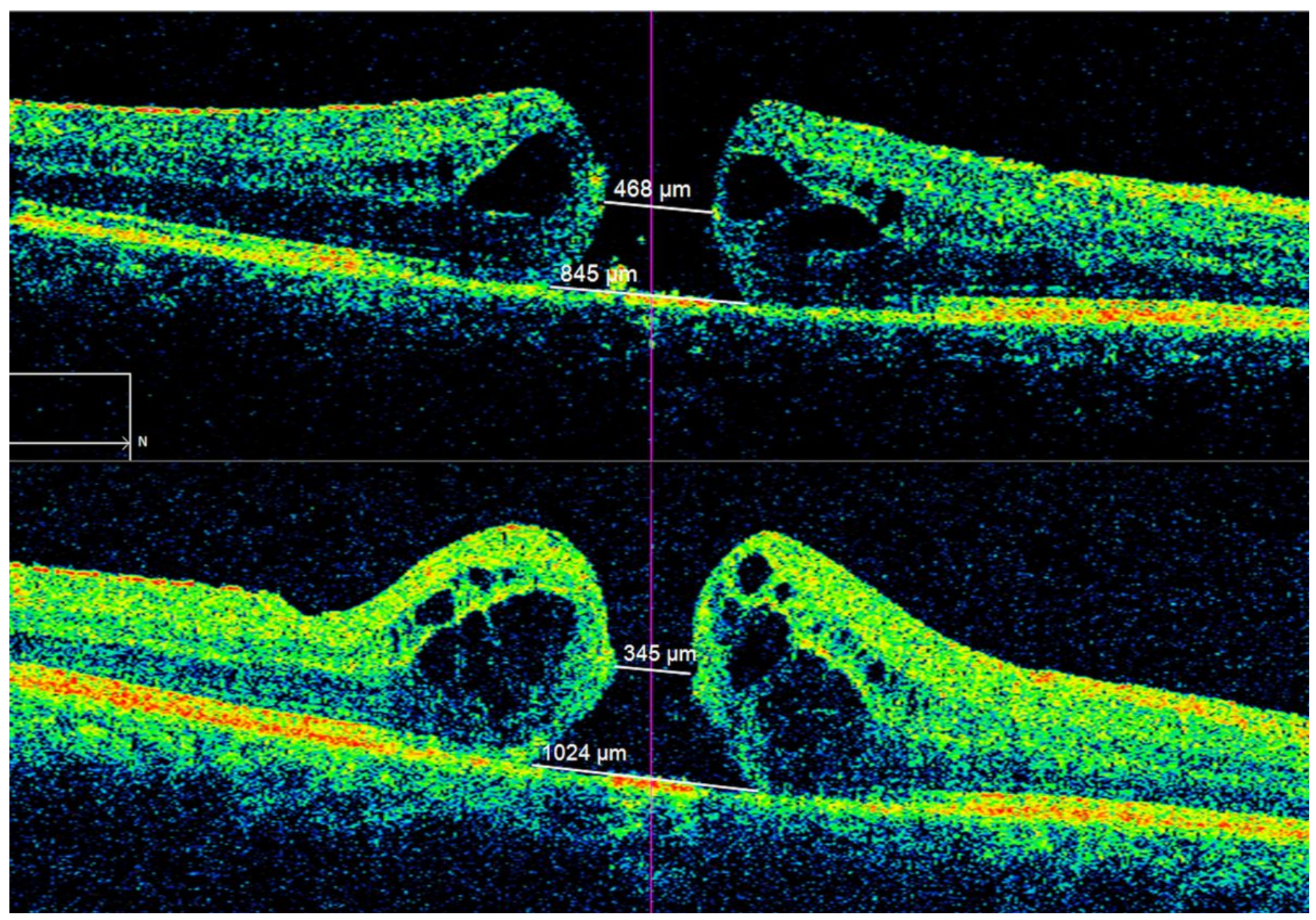

Figure 3

This is a 69-year-old man with a " $\mathrm{H}$ " type IMH. The minimal diameter was 468um and maximum diameter was 845um. The diameter of his MH was "small" but the HDR was more than 0.6. The MH was unclosed 3 months after PPV combined with ILM peeling and room air tamponade. 